\title{
Los sistemas de calefacción doméstica de la villa romana La Olmeda, Palencia
}

\section{The domestic heating systems of the villa romana La Olmeda, Palencia}

\author{
C. Gamarra ${ }^{(*)}$, J. A. Abásolo ${ }^{(*)}, \underline{\text { M. S. Camino }}{ }^{(*)}$
}

\section{RESUMEN}

El uso de hypocausta en estancias de uso no termal ha sido poco estudiado, de hecho, en casi todas las ocasiones se asocia al balnea doméstico de las villas o a las termas públicas. Pero también es frecuente en las villas romanas la existencia de hypocausta que proporcionan acondicionamiento térmico a dependencias no necesariamente relacionada con los baños de la villa.

En La Olmeda algunos de ellos efectivamente forman parte de la instalación termal del circuito de baños, pero la mayor parte de los que se han descubierto constituyen únicamente un sistema calefactor doméstico, muy necesario, por otra parte, en estas tierras de inviernos largos y fríos.

El objetivo de este artículo es el estudio de los mismos, su descripción y posible evolución en época romana, la planimetría de cómo pudieron estar constituidos y una aproximación a su funcionamiento. Del análisis de los datos obtenidos se ha llegado a la conclusión de que las características y los sistemas constructivos de los hypocausta están en relación con el momento de su concepción dependiendo de si se trataba de una nueva implantación o de alguna de las reformas debidas a la propia evolución de la villa romana.

Palabras clave: hypocaustum, arquitectura romana, bajo imperio, villa romana.

\section{ABSTRACT}

The use of hypocausta in non-thermal rooms has been little studied, in fact, in almost all occasions it is associated with the domestic balnea of the villas or the public baths. But it is also frequent in the Roman villas the existence of hypocausta that provide thermal conditioning to dependencies not necessarily related to the baths of the villa.

In La Olmeda some of them are indeed part of the thermal installation of the bath circuit, but most of those that have been discovered constitute only a domestic heating system, much needed, on the other hand, in these lands of long and cold winters. The objective of this article is the study of them, its description and possible evolution in Roman times, the planimetry of how they could be constituted and an approximation to its functioning. From the analysis of the obtained data it has been concluded that the characteristics and the constructive systems of the hypocausta are related to the moment of its conception depending if it was a new implantation or some of the reforms due to the own evolution of The Roman villa.

Keywords: Roman architecture, Low Roman Empire, Roman villa.

(*) Universidad de Valladolid.

Persona de contacto/Corresponding author: mcamino@arq.uva.es (M. S. Camino)

ORCID: http://orcid.org/oooo-00o2-2267-1601 (C. Gamarra); http://orcid.org/oooo-0oo2-7172-698X (J. A. Abásolo); http://orcid.org/oooo-0oo1-5711-3143 (M. S. Camino)

Cómo citar este artículo/Citation: C. Gamarra; J. A. Abásolo; M. S. Camino. (2018). Los sistemas de calefacción doméstica de la villa romana La Olmeda, Palencia. Informes de la Construcción, 70(550): e256. https://doi.org/10.3989/id.56900

Copyright: (C) 2018 CSIC. Este es un artículo de acceso abierto distribuido bajo los términos de la licencia de uso y distribución Creative Commons Reconocimiento 4.o Internacional (CC BY 4.0). 


\section{INTRODUCCIÓN}

Durante el Bajo Imperio hubo un desarrollo extraordinario de villas romanas en la región del Duero, fueron construidas "ex novo" algunas tan conocidas como La Olmeda, Almenara o Cuevas de Soria, pero también, Aguilafuente, Los Quintanares, La Tejada, Baños de Valdearados y otras. La Olmeda, situada al norte de la provincia de Palencia, se puede considerar la más destacada de todas ellas por su gran tamaño, su ordenada configuración de doble simetría (figura 1) y sus excepcionales mosaicos, algunos de los cuales podrían estar entre los mejores producidos durante el Imperio Romano. Se hallaba ubicada en las proximidades de la ciudad hispanorromana de Saldania, y a una veintena de km de Lacobriga o Viminacium por dónde discurría la calzada romana del "Norte", todas ellas civitates entorno a La Olmeda.

Los materiales hallados en La Olmeda, principalmente las monedas encontradas bajo el mosaico, permiten datar la villa a partir de mediados del siglo IV cuando Constancio II era emperador de los romanos (337-361). También por las monedas se puede conjeturar el máximo apogeo y el abandono de la villa. Parece que tanto el material numismático como el cerámico permiten estimar que la villa estuvo ocupada hasta mediados del siglo V (1).

La Olmeda se organiza de forma ordenada alrededor de un peristilo donde se disponen la mayoría de las dependencias de la residencia señorial. Todo el conjunto se haya orientado según dos ejes ortogonales que se cortan en el centro del peristilo. El eje norte-sur es el más relevante, presentando casi una simetría axial en los ámbitos norte y sur dónde se encuentran sendas entradas a la villa, manifestándose también en sus fachadas simétricas, formadas respectivamente por galerías porticadas de dos plantas y enmarcadas por sendas torres, octogonales al sur, dónde se halla el acceso principal, y sensiblemente cuadradas al norte. Por su parte el eje esteoeste presenta en su extremo oriental la cámara más importante y de mayor tamaño de la villa como es el oecus, siendo el contrapunto en el área occidental todo el complejo termal de casi $900 \mathrm{~m} 2$ que se dispone a modo de apéndice apoyado y a ambos lados de un enorme corredor que alcanza el peristilo. El conjunto termal de La Olmeda está considerado entre los más monumentales de las villas de Hispania (2).

En las excavaciones de la villa se han encontrado restos de hypocausta tanto en la zona termal como en otras habitaciones importantes de la misma. El objetivo de este artículo es precisamente el análisis de los hypocausta que se utilizaron para caldear las habitaciones ajenas al circuito termal.

\section{EL HYPOCAUSTUM COMO SISTEMA DE CALEFACCIÓN}

La palabra hipocaustum procede de otro término griego que significa "fuego por debajo", en realidad, fue reinterpretado por los romanos que ya desde el siglo II a.C. (3) comenzaron a utilizarlo. Este sistema de calefacción se fundamentaba en una cámara horizontal subterránea (4) que se combina con la tubulatura (5), o calefacción vertical, basado en una doble pared con cámara hueca o conductos, comunicados con el hipocausto, con lo que simultáneamente se calentaban el suelo y las paredes de la estancia. Si bien en los hypocausta que

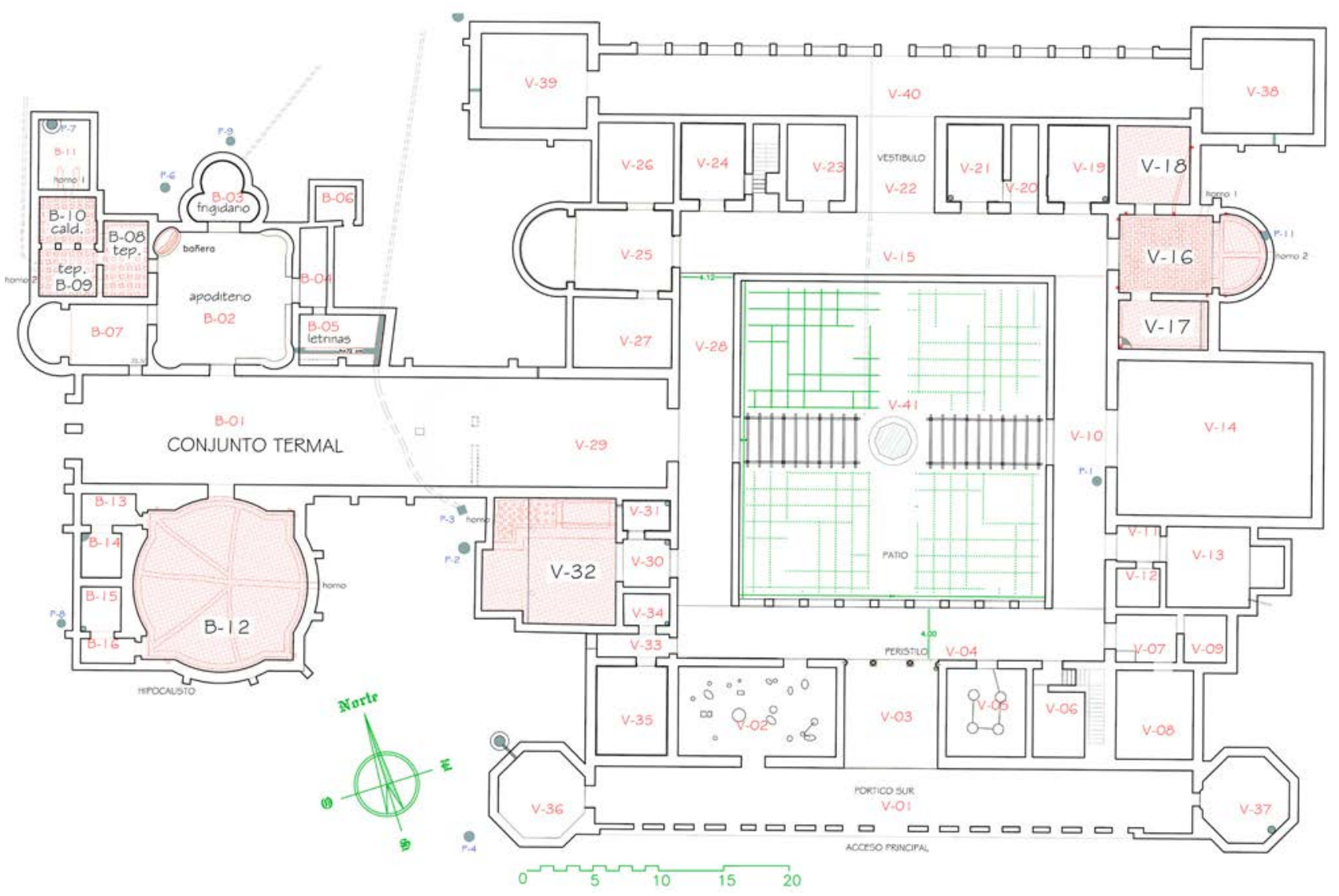

Figura 1. Planta de La Olmeda (C. Gamarra). 
estudiamos en este artículo no se han localizado indicios del empleo de tubulatura o concamerationes.

En general, el hypocausis (6) se asocia con las termas romanas pero en realidad es un sistema de calefacción que igualmente podía calentar un comedor o una sala de reunión. En el praefurnium la combustión de madera o carbón vegetal generaba gases y aire caliente que se distribuía por la cámara de calor bajo el pavimento o los conductos verticales de los muros. A partir ahí, la configuración específica de cada hypocaustum podría tener distintas peculiaridades, relativas al horno, a la cámara de calor del suelo a los conductos en las paredes o a la evacuación de los humos.

La construcción de la cámara de calor bajo el pavimento es el elemento más representativo del hypocaustum y podía hacerse de diversas formas, siendo la citada por Vitrubio la más conocida y en la que el piso elevado o suspensura de los baños se construye sobre "unos pilares de pequeños ladrillos de ocho pulgadas, teniendo en cuenta que se puedan intercalar en medio unas tejas de dos pies; la altura de los pilares será de dos pies" (7). Los pilae podrían ser cuadrados, rectangulares o circulares, de ladrillos o de piedra, se pueden encontrar arquillos sobre los pilae, o ladrillos cuadrados bipedales, en vez de tégulas, que apoyan en sus cuatro esquinas sobre los respectivos pilae.

Una variante de mayor calado en los hipocausta es que la suspensura no apoye sobre pilares sino sobre muretes de albañilería formando diversos canales o conductos comunicados entre sí por los que transita el aire caliente. Los canales pueden ser lineales rectos o curvos, lo más usual son varios canales paralelos, o también pueden ser radiales como en el caso de Silchester (Reino Unido) (8). Si en el primer caso, el hipocaustum clásico, el subsuelo de la habitación está prác- ticamente hueco, en el segundo está más bien macizado y los únicos huecos son los canales citados.

En la villa de La Olmeda se han encontrado los dos sistemas diferentes indicados: unos realizados con pilae de ladrillos y otros de canales que en ocasiones son paralelos, otras veces se adaptan a la forma circular de la habitación o son radiales partiendo del centro de la habitación, de manera muy similar al de Silchester. En un artículo sobre los hypocausta de La Olmeda resulta obligado mencionar los de una villa muy cercana, como es La Tejada, en Quintanilla de la Cueza. Hoy los investigadores dudan de su consideración como villa, pero de lo que no existe ninguna duda es que cuenta con un soberbio conjunto de hypocausta. Entre ellos son curiosos los de las habitaciones 12 y 18 que con un gran canal central y otros paralelos y ortogonales al anterior serían del segundo tipo de los anteriormente mencionados pero que se aproximan al clásico porque el conjunto acaba ocupando toda la superficie de las habitaciones respectivas.

En la construcción de hypocausta se utilizaron diversos materiales como ladrillo, piedra, incluso la propia tierra (9). El ladrillo (10) fue, sin duda, el más usado y así se ha podido constatar en algunas de las villas del Duero, tales como La Tejada, Almenara, Cuevas de Soria y en La Olmeda, dónde además fue muy común en las infraestructuras de suministro de agua, alcantarillado, así como en diversos sistemas constructivos de los muros de la villa (figura 2).

Los constructores romanos conocían el excelente comportamiento del ladrillo ante el fuego, el agua y su resistencia mecánica (11). Con piezas de ladrillo se hacían diversos tipos de canales, los pilae, incluso la suspensura con varias capas de ladrillos o fragmentos de tegulae trabados con mortero de cal. El ladrillo es un material con gran inercia térmica y por

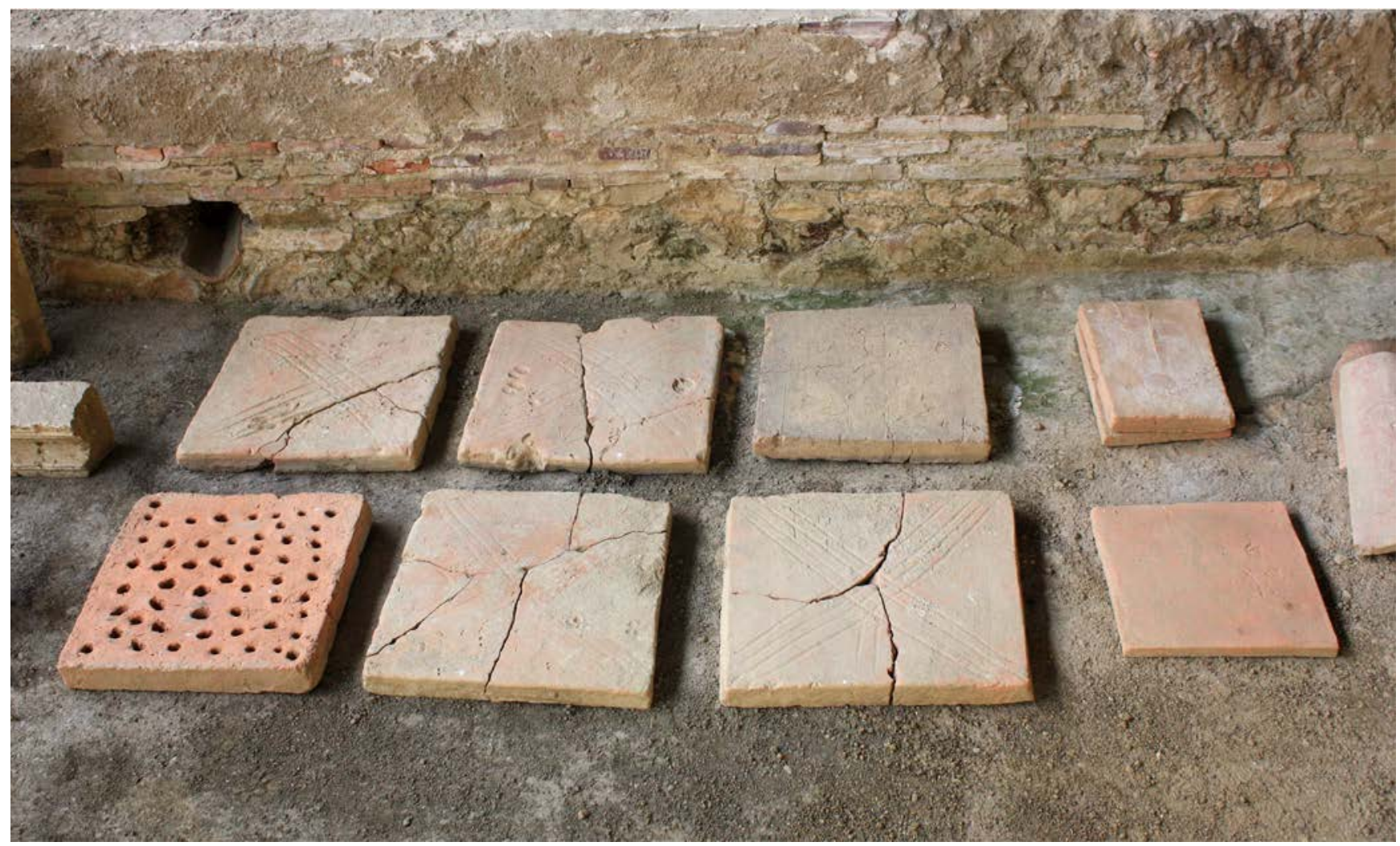

Figura 2. Algunos tipos ladrillos encontrados en la villa (C. Gamarra). 
ello posee gran capacidad para almacenar calor que después va irradiando lentamente caldeando la habitación superior.

El ladrillo era un material apreciado que podía tener un segundo uso y cuando la villa entraba en una fase decadente y era abandonada casi siempre acababa por producirse el expolio de la misma para recuperar este material. Lamentablemente al levantar el hypocaustum este quedaba destruido y con ello también el mosaico situado encima (figura 3). Aunque este expolio permita conocer como estuvieron construidos los hypocausta.

\section{DESCRIPCIÓN Y LOCALIZACIÓN DE LOS HYPOCAUSTA ANALIZADOS DE LA VILLA DE LA OLMEDA}

Los hypocausta a estudiar son los de las habitaciones V-16 y $\mathrm{V}-32$ que se hallan localizadas respectivamente al noreste y suroeste del patio interior y el de la habitación B-12 situada al sur del área termal, pero que no forma parte del circuito de baños Las tres habitaciones citadas eran piezas de prestigio y representación social, con gran amplitud aunque de diferente tamaño, y con formas singulares. Todas ellas podrían destinarse a actividades públicas como era el recibir a la clientela, visitantes y amigos.

La V-16 disponía de un ábside, por lo que muy bien podría tratarse de un triclinio. Se trata de una estancia excavada en 1983, de la que se conocía su existencia porque los praefurnia se habían localizado unos años antes. Además, esta pieza $\mathrm{V}-16$, forma con las colindantes, V-17 y V-18, una especie de grupo de habitaciones, con las que se comunica y a las que extiende su sistema de calefacción, que confiere a este blo- que de estancias un valor añadido, que no posee su simétrico. Podrían ser dependencias para uso directo del dueño y su familia, una especie de sala de estar o un comedor, o cualquier otro uso de prestigio; y las piezas contiguas destinadas a actividades complementarias, por ejemplo, alcobas para la familia o invitados ilustres.

La habitación V-32 y toda la zona adyacente del suroeste de la villa, entre el vestíbulo de entrada, V-03, y el acceso a los baños, V-29, fue excavado entre 1987 y 1990 (12). Los dos directores que han tenido las excavaciones consideran que esta gran pieza con exedra rectangular es un comedor o triclinio (12-13). Los restos conservados de muros, mosaicos e hypocausta parecen indicar que hubo distintas etapas en su configuración. Los objetivos que se pretendían en esa supuesta evolución eran aumentar su superficie y dotarla de un sistema calefactor. La habitación se amplía hacia el norte ocupando una cámara previsiblemente de servicio y, por ello, es precisamente en esa zona dónde se construye el hypocaustum. La ampliación supone que la exedra existente no está centrada con el nuevo ámbito rectangular, y que se halle formada por dos alfombras de mosaico diferente, la más antigua y la ampliada.

En 1994-95, tras la construcción de una edificación que protegía un área en torno a los baños, se excavó la estancia B-12, consolidándose y restaurándose en los años siguientes. Además del hypocaustum es destacable su original planta central con su excepcional tamaño y el pavimento de mosaico geométrico complejo con el tema de scuta (escudos) (14). Algunos investigadores situaron en ella el apoditerio (15), y no es descartable que en algún momento hubiera podido tener ese uso, aunque fuera como apoyo de la otra gran pieza de

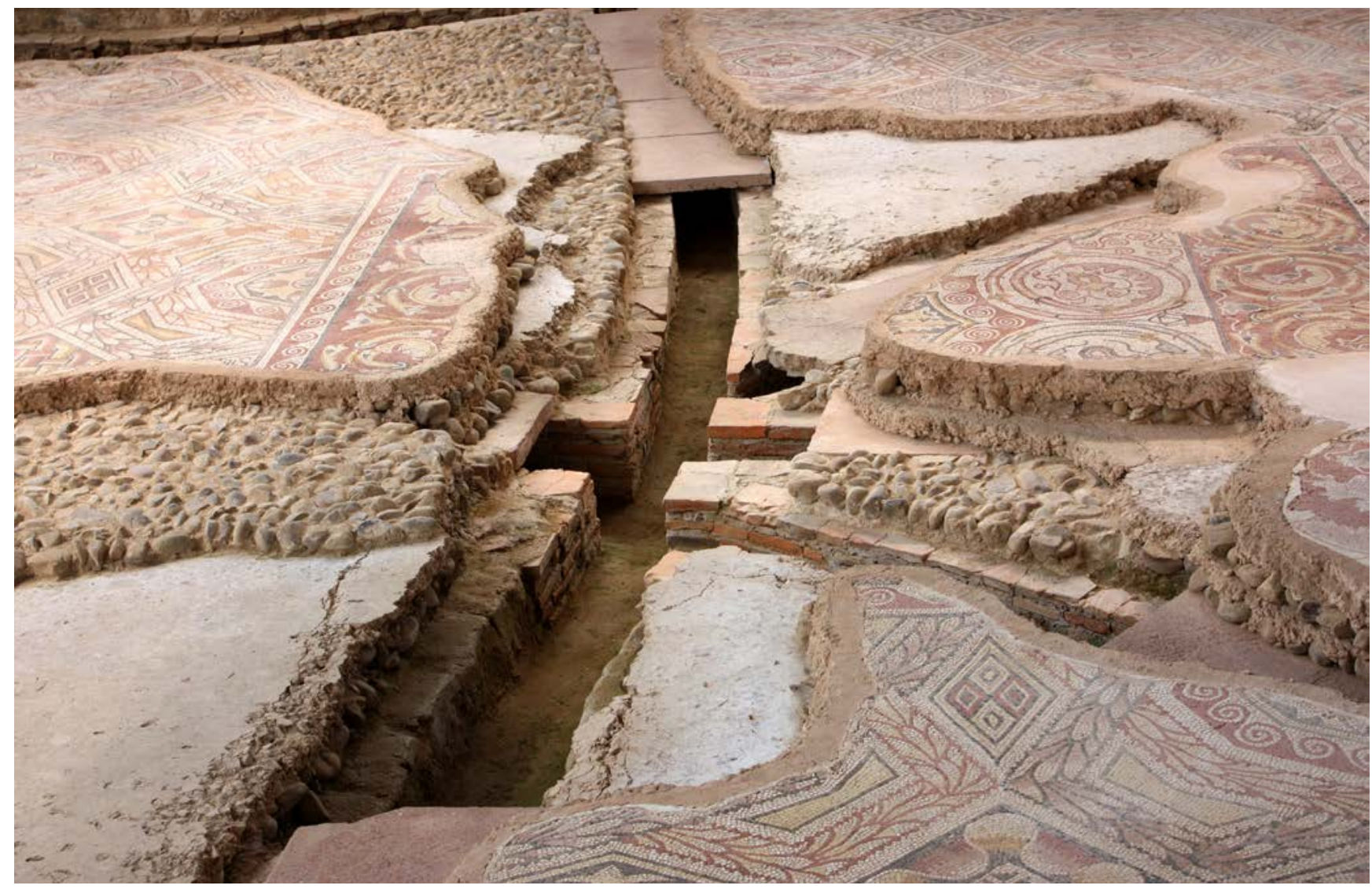

Figura 3. Habitación B-12, destrucción producida al recuperar los ladrillos (C. Gamarra). 
los baños, B-02, que con su banco corrido y proximidad a las cámaras frías y calientes fuera más propiamente el apoditerio-frigidario. Habitaciones de honor similares a esta en el área de los baños, aunque no tan bien dotadas como la de La Olmeda (con mosaico e hypocaustum) también están presentes en otras villas romanas contemporáneas cercanas como Almenara, Quintanares o Aguilafuente.

En la figura 1 se indican todas las habitaciones descritas anteriormente con presencia de hypocausta no termales que constituían el sistema doméstico de calefacción en la villa (también se indican las destinadas a los baños domésticos a título orientativo, V-08, V-09 y V-10). Para su estudio se han levantado los planos que se incluyen en este artículo y se han analizado los restos conservados que proporcionan información del último período de habitación y, en ocasiones, de una cierta evolución a lo largo de su tiempo de uso (1).

\subsection{Hypocausta en la habitación V-16, V-17 y V18}

La habitación V-16, disponía de una superficie de 68,87 m2 y se hallaba organizada en dos partes bien diferenciadas, una rectangular de 7,13 x 5,98 m y otra semicircular, o mejor ultrasemicircular, unidas por una franja de $0,55 \mathrm{~m}$ entre dos contrafuertes que estrechan la habitación en la zona de unión (figura 4).

La habitación tenía calefacción con dos sistemas diferentes según los ámbitos, rectangular o absidal que estaban comunicados entre sí. En la zona rectangular existía una cámara con pilae organizados según los ejes ortogonales de la habitación (figura 5). De esta manera se forman 12 filas en sentido norte-sur de 11 pilae cada una. Excepto los de la primera fila junto al peristilo que tienen mayor sección, $48 \times 40 \mathrm{~cm}$ (16), el resto son ladrillos cuadrados de $32 \times 32 \mathrm{~cm}$ (algo superior al formato pedal de 29,6 x 29,6 cm).

Cada pilae tiene una altura de 7 hiladas de ladrillo y unos $40 \mathrm{~cm}$ de altura. La separación entre pilae es de unos $20 \mathrm{~cm}$ aproximadamente. En el perímetro hay un murete o zócalo de fragmentos de ladrillos y tejas con mortero de barro hasta el mismo nivel que los pilae. La suspensura, construida con dos capas de ladrillo cubriendo los conductos ortogonales, estaba cubierta por una capa de unos $10 \mathrm{~cm}$. de opus signinum sobre la que se disponía el mosaico del pavimento. Por la cámara de calor creada bajo el suelo circulaba horizontalmente el aire caliente procedente de un horno situado en el exterior de la habitación en el extremo oriental del muro norte, a través de un orificio de $42 \times 30 \mathrm{~cm}$. Se hallaba limitado por un contrafuerte coincidente con el contrafuerte interior septentrional de la exedra.

Para facilitar las salidas de humos de la cámara del hypocaustum se interrumpe en diversos puntos el zócalo perimetral alcanzando unas perforaciones verticales en los muros (chimeneas) o en otros casos configurando unas canalizaciones a las habitaciones colindantes. Se conservan dos de estas salidas verticales en cada uno de los tres muros que delimitan el hypocaustum, oeste, sur y norte. Son sensiblemente cuadradas y separadas del espacio interior por una placa de tégula. Las del muro sur pasan también horizontalmente a la pieza colindante V-17, y bordeándola van a parar al ángulo suroeste, dónde algunos investigadores sitúan una gran chimenea (16). Algo parecido ocurre con la salida situada a unos $2 \mathrm{~m}$ de la boca de enroje en el muro norte que continuaría además bajo el mosaico de la habitación V-18 hasta otra previsible salida de humos próxima a la esquina noreste de esa habitación.

Este hypocaustum se comunicaba con el de la exedra por tres huecos triangulares de ladrillo por delante del ábside y el muro de piedra adosado que separa los dos hypocausta, repitiéndose unos huecos idénticos.

El hypocaustum de la exedra constaba de un sistema de diversos canales adaptados a su geometría semicircular por dónde pasaba el aire caliente: uno de las cuáles es un conducto en dirección norte-sur coincidente con el diámetro de la exedra; otro, perpendicular al anterior por su centro, coincidente con

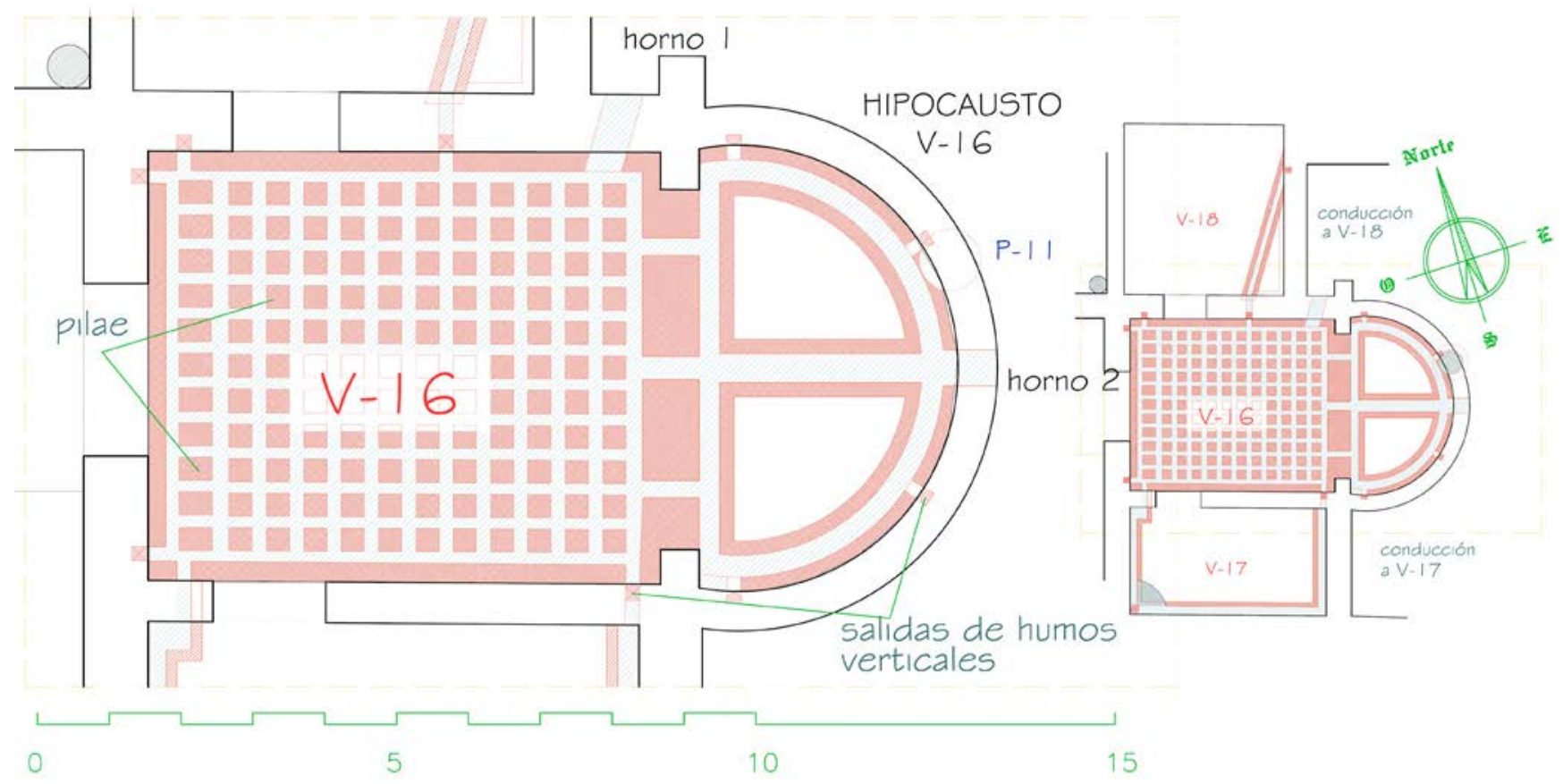

Figura 4. Hypocaustum de V-16 y colindantes (C. Gamarra). 


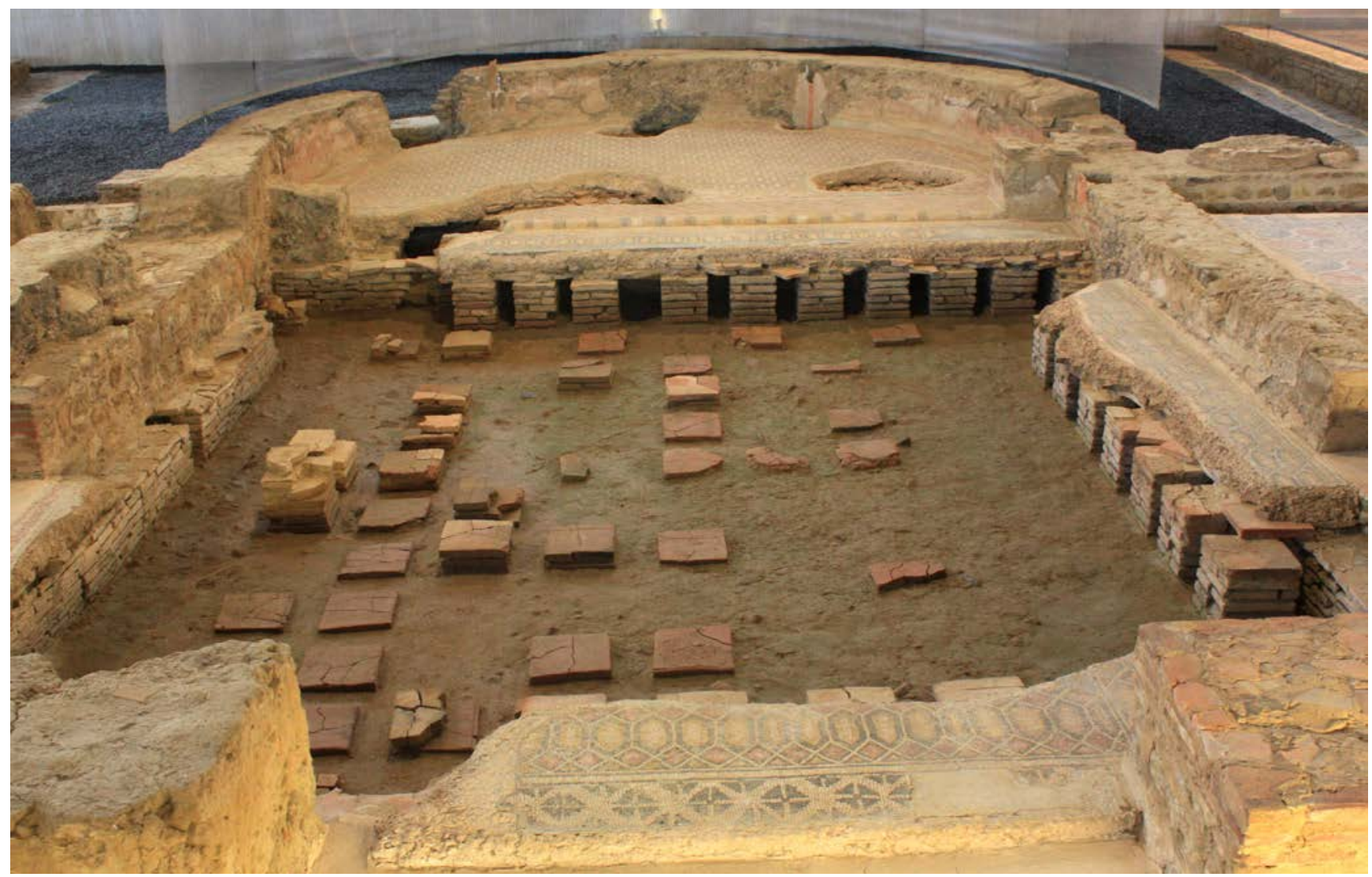

Figura 5. Vista del hipocausto de V-16 (C. Gamarra).

el eje longitudinal de la habitación, en sentido este-oeste; y un tercero que la circunda perimetralmente. Estos canales tienen una anchura de 30-40 cm, una profundidad aproximada de 45-50 cm y se van estrechando según aumenta la altura para favorecer la colocación de la losa superior. Los canales están realizados con hileras de ladrillos o fragmentos de éstos cogidos con barro. Al igual que en la zona rectangular, sobre los conductos, se superponen dos hiladas de baldosas, tejas o ladrillos y una gruesa capa de opus signinum.

El hypocaustum de la exedra tiene su propia entrada de fuego, en el muro curvo de la exedra, coincidiendo con el eje longitudinal de la habitación, mediante un orificio de $45 \times 45$ $\mathrm{cm}$. Hay cuatro chimeneas o perforaciones distribuidas más o menos uniformemente a lo largo del muro semicircular de la exedra. Son simples orificios de unos $17 \mathrm{~cm}$, protegidos en la cara interna por tejas ímbrices.

Toda la habitación estuvo pavimentada con mosaico a base de hexágonos irregulares yuxtapuestos por dos de sus lados alargados y formando cuadrados con los cortos. Se conserva una buena parte del situado en el ábside y pequeños fragmentos testimoniales en la zona rectangular. Entre los dos sectores hay un ligero desnivel situándose la exedra unos $5,5 \mathrm{~cm}$ más alta, que se salva con una especie de pequeño escalón, de cuarto de círculo, cubierto con un mosaico de bandas paralelas.

\subsection{Hypocausta en la habitación V-32}

El área calefactada se localizaba junto al acceso a los baños, V-29, (Figura 6), con un mosaico diferente del resto de la cámara. El hypocaustum tuvo seguramente su origen en el desarrollo de diversas ampliaciones de esta estancia y quizás por ello cuenta con dos sectores diferentes, incluso con diferencias apreciables entre ellos.

La superficie real de la habitación es de 92,00 m2, pero el centrado de la exedra pudo generar algunos espacios residuales que reducirían la superficie del mismo hasta unos 78,45 m2 -excluyendo los espacios C y D- (figura 6). Aun así, tiene aproximadamente una superficie doble que el triclinio que podría ser considerado más familiar en la esquina sureste (V13). Por ello, debió ser el comedor de los grandes banquetes con los que el dominus obsequiaría a sus clientes y amigos de la élite hispanorromana.

El sector oeste (I) (Figura 7) se configura como una cámara con pilae, de unos 20 x $20 \mathrm{~cm}$-ladrillos bessalis- y con una altura de unos $50 \mathrm{~cm}$. La forma del hypocaustum es sensiblemente rectangular aumentando sus dimensiones con un pequeño apéndice junto a la entrada del praefurnium en el borde occidental: longitud este-oeste 4,43 m; y norte-sur 1,82 $\mathrm{m}$ (2,93 $\mathrm{m}$ junto al praefurnium); se considera la distancia libre entre apoyos del zócalo perimetral.

En general, la disposición de los pilae se adapta a dos geometrías diferentes (figuras 6 y 8), que han tenido que ver con las ampliaciones del hypocaustum de forma paralela a las transformaciones del triclinio. Una de las geometrías está formada por pilae de ladrillos organizados según filas y columnas ortogonales según los ejes de la habitación, que van alternando en la mayor parte de esta área en posiciones intermedias con las filas y columnas de la otra geometría, en este caso con los pilae a $45^{\circ}$. El sector I está directamente en contacto con un hueco en el muro de 50 x $44 \mathrm{~cm}$ para el enroje del hypocaustum (figura 7). 


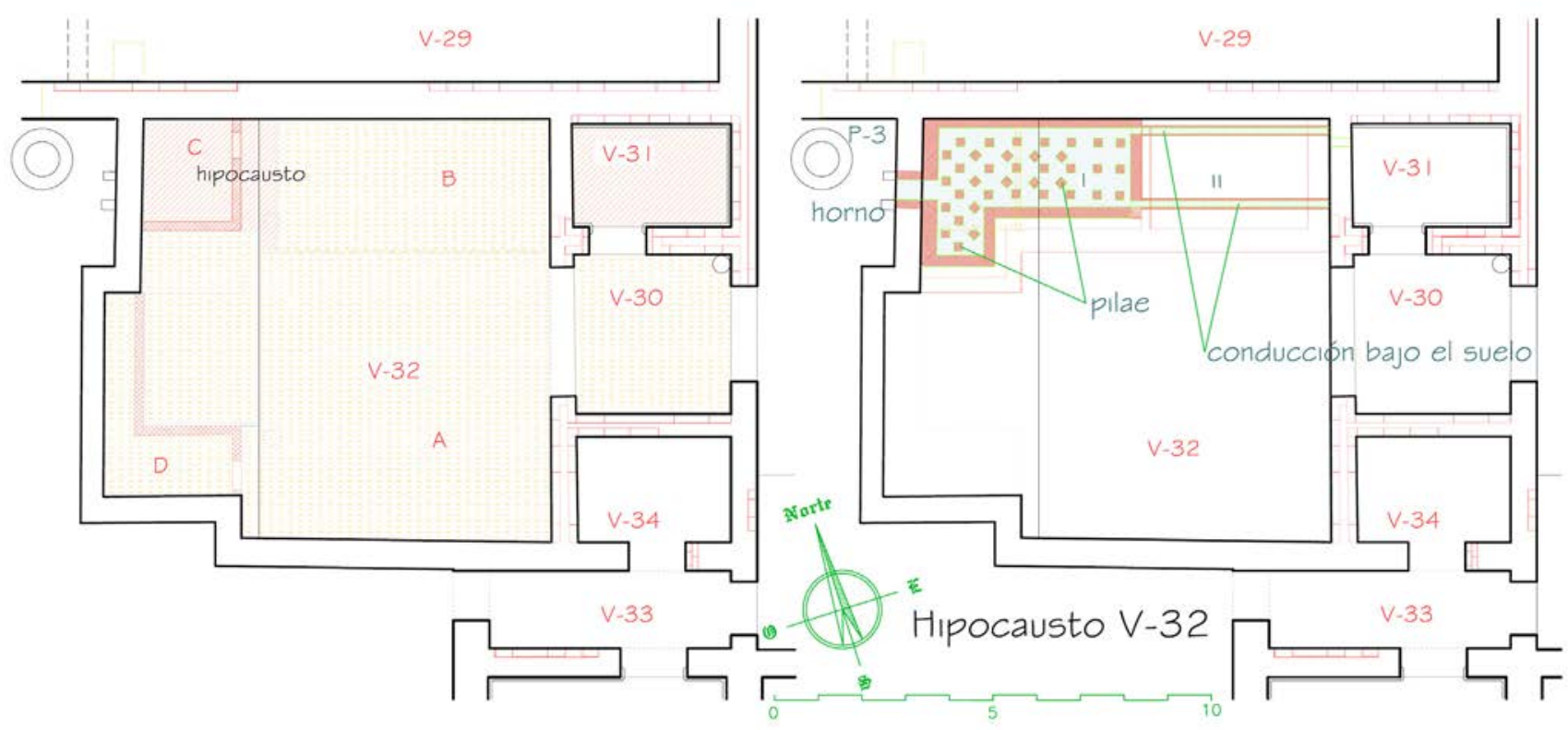

Figura 6. Planta de la habitación V-32 (C. Gamarra).

El sector oriental del hypocaustum (II) tiene dos canales paralelos en dirección este-oeste de unos 4,60 m de longitud y separados 1,65 m (figura 6) que llegan a alcanzar el muro que separa esta habitación con la colindante al este, V-31. Los conductos tienen una sección aproximada de $14 \mathrm{~cm}$ de ancho por $40 \mathrm{~cm}$ de altura.
El sector occidental construido sobre pilae se encuentra prácticamente arrasado. El deterioro impide una mejor interpretación del área, pudiéndose observar el hueco de la cámara de gases calientes, unos pocos pilae de los dos tipos especificados, y en algún punto la suspensura a base de dos placas de ladrillo o tégula y losa de mortero de cal de unos 10-12 cm (figura 8).

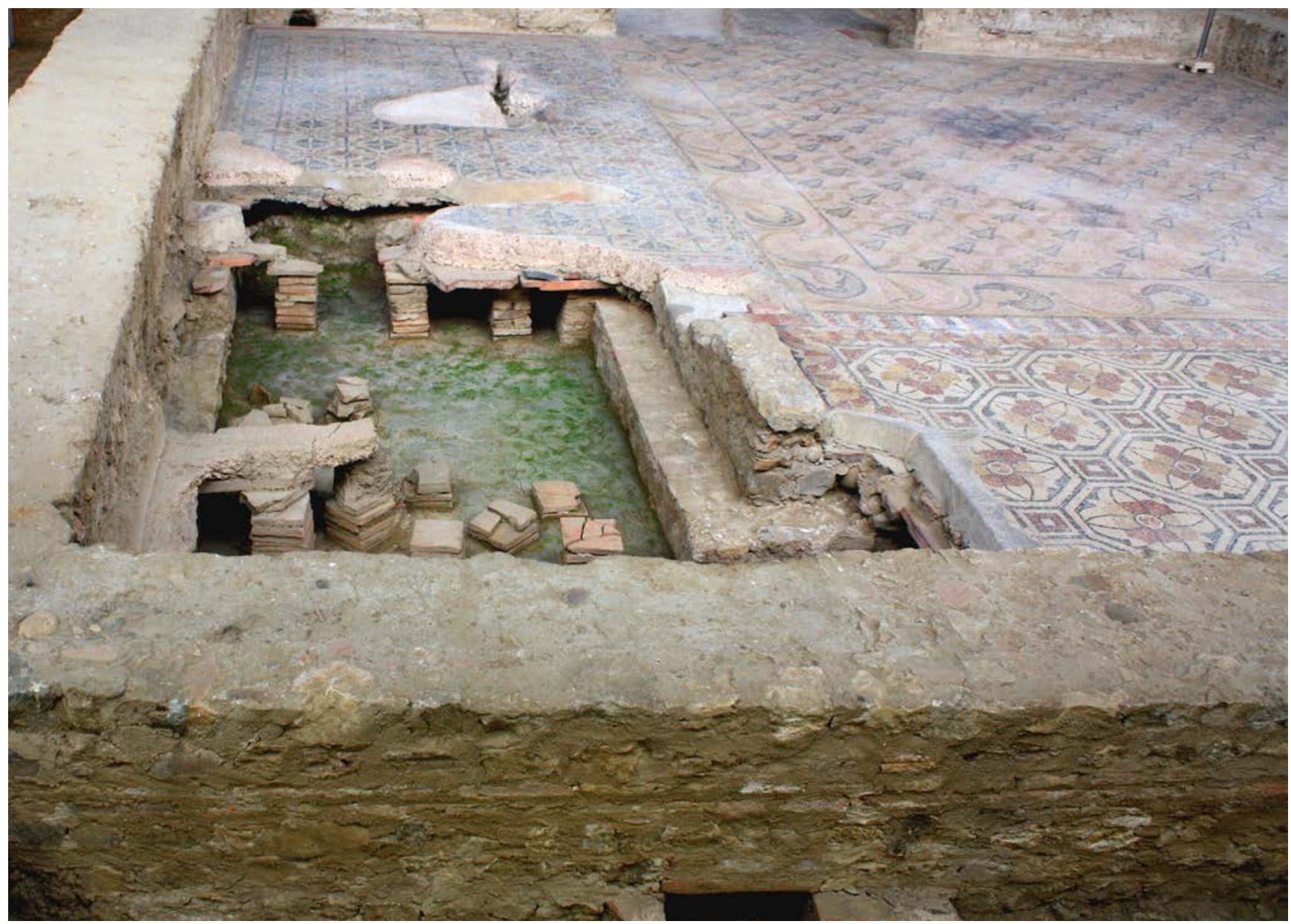

Figura 7. Vista del hipocausto desde el exterior oeste de la cámara (C. Gamarra). 
Sin embargo, el sector oriental, mucho más simple y oculto de cara a los furtivos, se conserva en unas aceptables condiciones con la mayor parte del pavimento de mosaico sobre las dos canalizaciones, aunque también con algún que otro socavón, producto de los agujeros que practicaban para avistar los ladrillos.

Como todas las piezas relevantes de La Olmeda esta habitación estaba pavimentada en su mayor parte con mosaico. En este caso se traduce en varios diseños que se corresponden con distintas etapas de su configuración. En el área de mayor superficie del primer triclinio, señalado como "A" (figura 6), el dibujo del mosaico consiste en círculos amarillos exentos unidos por eslabones rojos y tallos en diagonal que partiendo del centro de los círculos acaban en una especie de tres pétalos con forma de campanillas. El mosaico ampliado de esta zona, señalado como "B" (figura 6), es muy diferente con una malla reticulada con círculos exentos y motivos florales.

También hay dos diseños con cierta similitud para las dos exedras que tuvo el comedor a lo largo de su período de uso. El pavimento de la última se conserva casi íntegro, ya que se halla superpuesta y ligeramente desplazada sobre la primera, lo que ha permitido también conocer el diseño de la más antigua al haberse conservado una pequeña superficie en forma de "L" (señalado como D en la figura 6; detalle en la figura 9). En ambos casos y aunque con distintas proporciones se trata de un dibujo de octógonos irregulares yuxtapuestos por los lados más largos y formando cuadrados con los más cortos.

\subsection{Hipocaustum de la habitación B-12}

La habitación B12 presenta una forma peculiar de planta central derivada de la superposición de un círculo y un cuadrado también definida como planta tetralobulada. El diámetro de la circunferencia es de unos $13,40 \mathrm{~m}$, y el lado del cuadrado de 11,40 m. La superficie total de la habitación es de unos 148,00 m2, la más grande de la villa después del oecus (figuras 10-12). La cámara se halla situada al sur del enorme pasillo que conduce a la vivienda y frente al apoditerio $\mathrm{B}-02$, la otra gran pieza importante de los baños, al otro lado del corredor.

El hypocaustum realizado a base de conductos es poco convencional, consta de seis canales radiales que se han denominado por su orientación con respecto a los puntos cardinales (E, N, NO, O, SO y S) y otro perimetral. Las conducciones no se hallan uniformemente distribuidas por la habitación, siendo más numerosas en la mitad oeste de la cámara. Esa mayor concentración de conductos se puede explicar por la intención de dotar al hypocaustum del tiro conveniente, evitando cambios bruscos de dirección en la salida de los humos de combustión.

Los canales radiales tienen una anchura de unos $30 \mathrm{~cm}$, excepto el de entrada o principal, que une el centro de la habitación con el praefurnium (E), que tiene unos $45 \mathrm{~cm}$. El perimetral circunda toda la habitación y tiene unos $40 \mathrm{~cm}$.

Los canales se excavaron en el terreno arcilloso y se construyeron con diversos materiales constructivos (17). La conducción perimetral este y sur fue realizada a base de muretes de mampuestos de piedra y cantos rodados; uno de los cuáles se adosa a la cimentación del muro de carga y forma un escalón de unos $22 \mathrm{~cm}$ de anchura, usual también en las otras paredes de los canales. Todos los cruces e intersecciones están construidos con hiladas de ladrillo sobre base de fábrica de piedra (figura 3).

Finalmente el resto de los canales, perimetrales o radiales, fueron construidos con varias hiladas de adobes de gran ta-

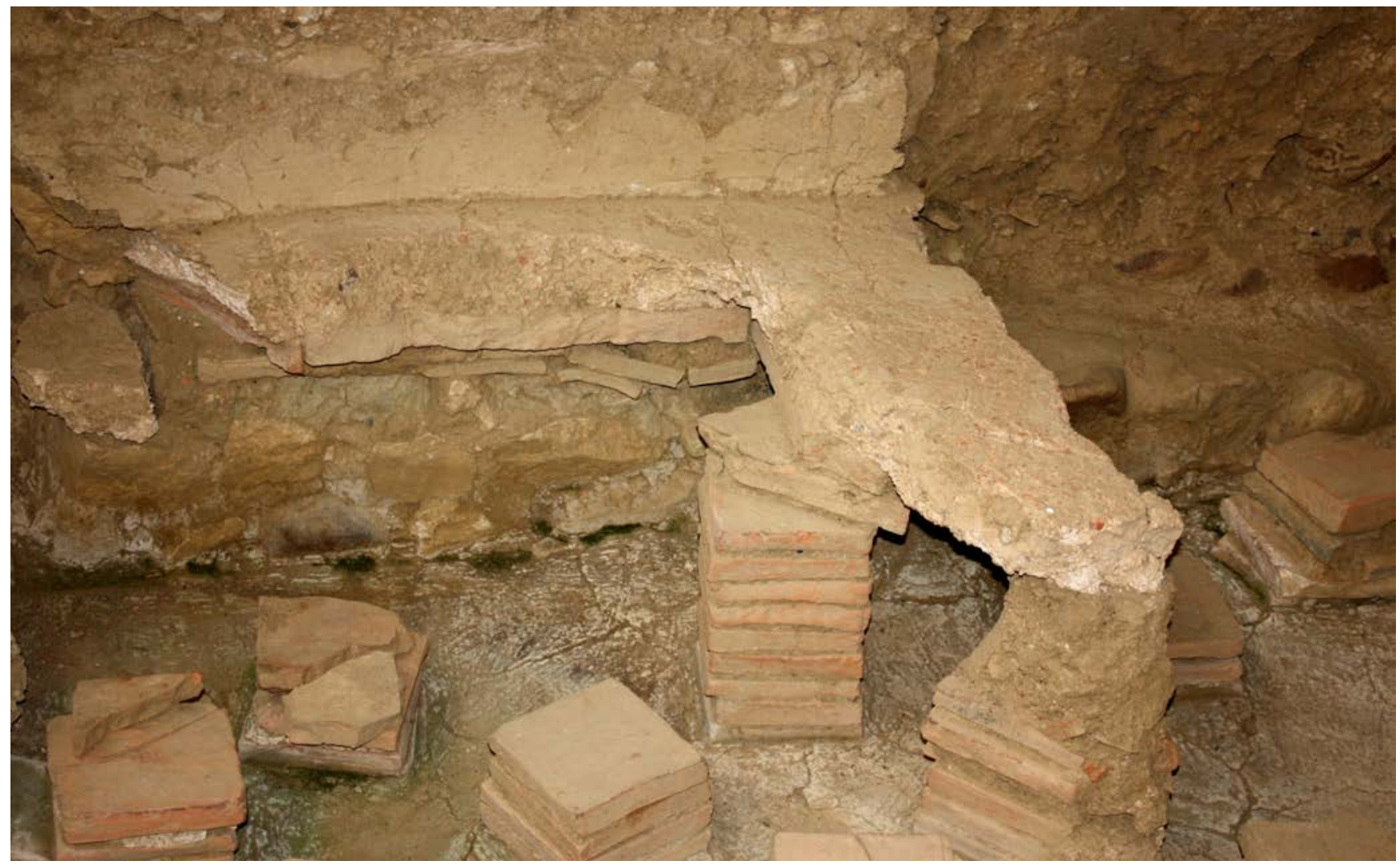

Figura 8. Restos de opus signinum sobre pilae (sector I) (C. Gamarra). 


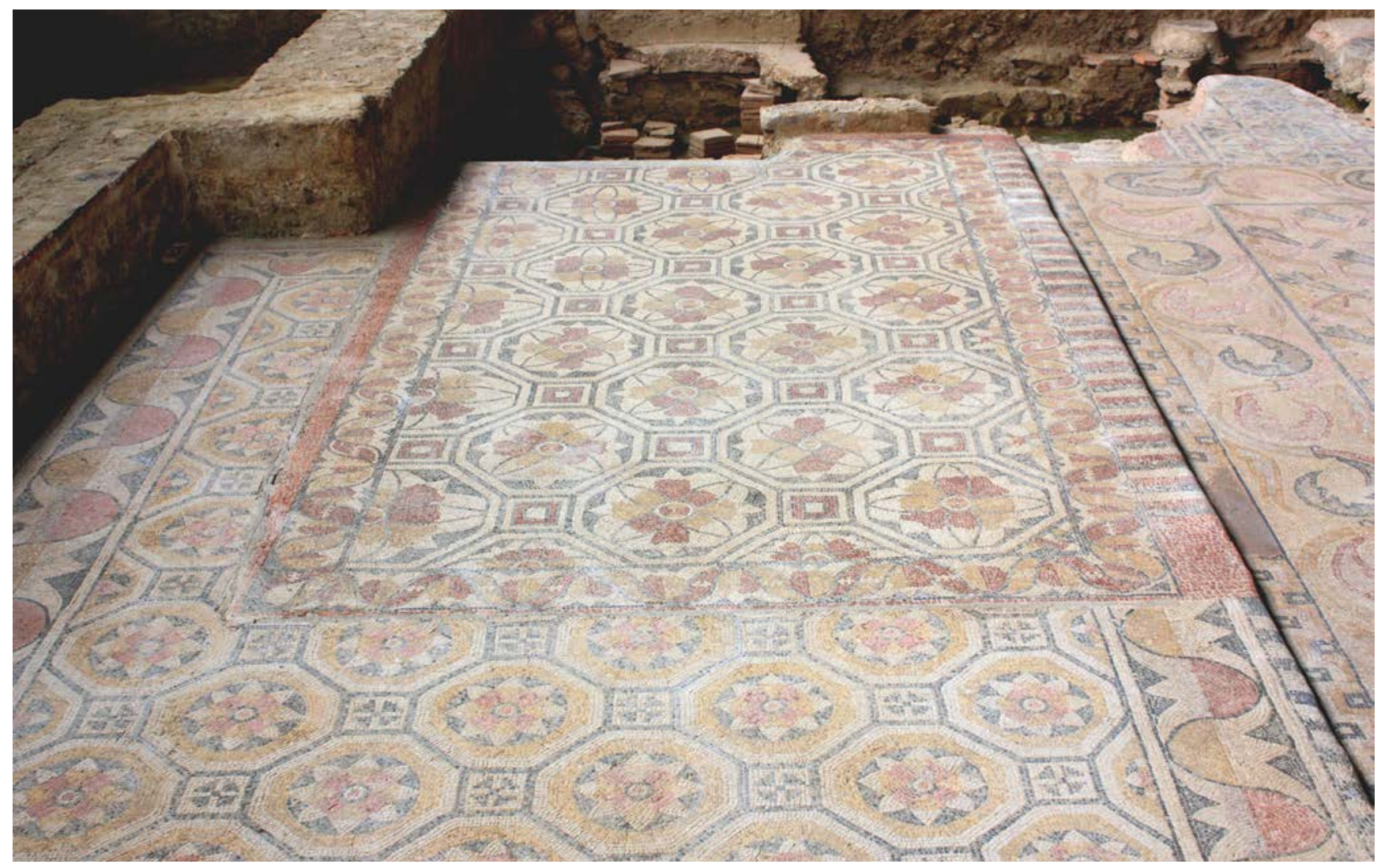

Figura 9. Solapamiento de los pavimentos de la exedra, al fondo el hypocaustum (C. Gamarra).

maño: 43 x 29 x $23 \mathrm{~cm}$. Evidentemente, no es apropiado el uso de adobes en contacto con el terreno, porque se deterioran con la humedad del mismo. Cuando se excavó había además mucha humedad y las paredes de adobes a duras penas eran perceptibles, pero había una excepción, la canalización norte $(\mathrm{N})$, donde se conservaban tres hiladas de adobes que permitieron constatar su aspecto original (18) (figura 3).

Los canales del hypocaustum estaban cubiertos por los ladrillos bipedales de 59,2 x 59,2 cm (en la figura 2 se pueden observar algunos de estos ladrillos) y sobre ellos el primer

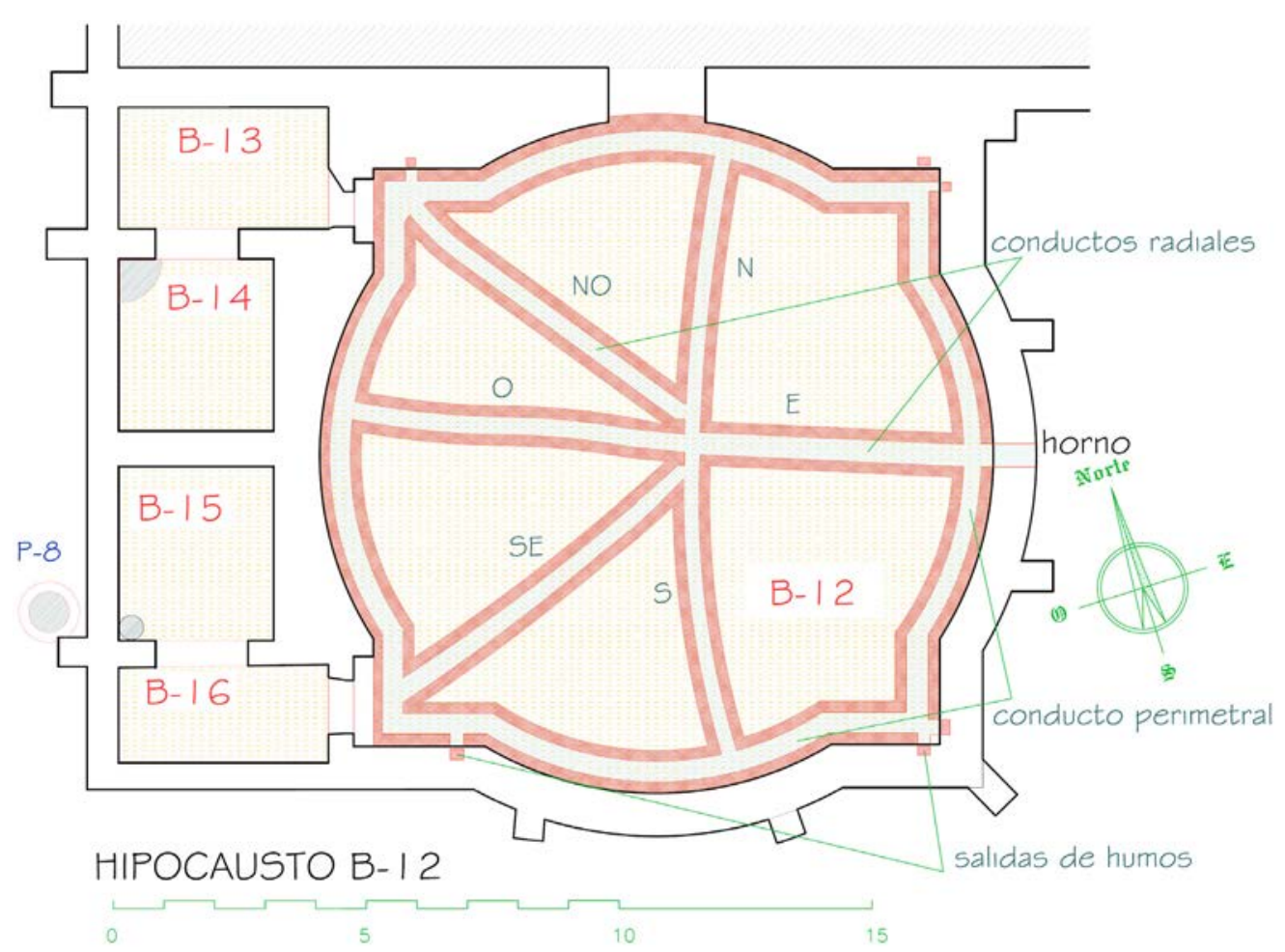

Figura 10. Hipocausto de la B-12 (C. Gamarra). 


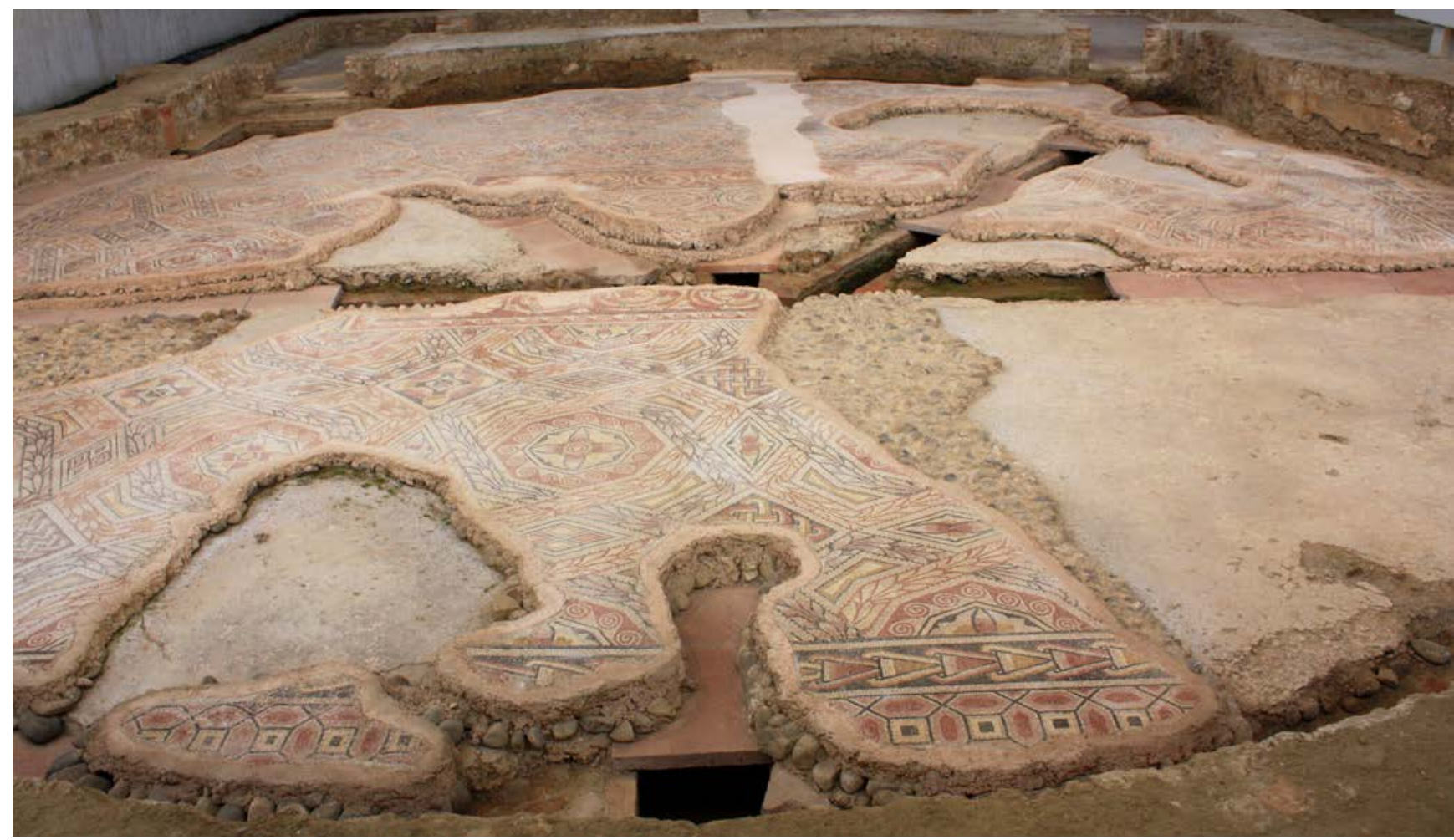

Figura 11. Vista general de la habitación desde el borde oriental (C. Gamarra).

pavimento de opus signinum de la habitación. El saqueo de estos ladrillos fue determinante en la destrucción de los suelos y mosaicos que cubrían la estancia. En la galería suroeste (SO) se encontraron cinco de esos grandes ladrillos, que tenían unos $6 \mathrm{~cm}$ de espesor (19). En algunas de las figuras exhibidas se puede observar la restauración del hypocaustum y el uso de unos "bipedales" con aspecto similar a los originales (figuras 11-12).

El praefurnium está situado al exterior de la habitación junto a la pared este. La boca formaba un arco de medio punto y cuando fue excavada apareció tapiada con fragmentos de teja

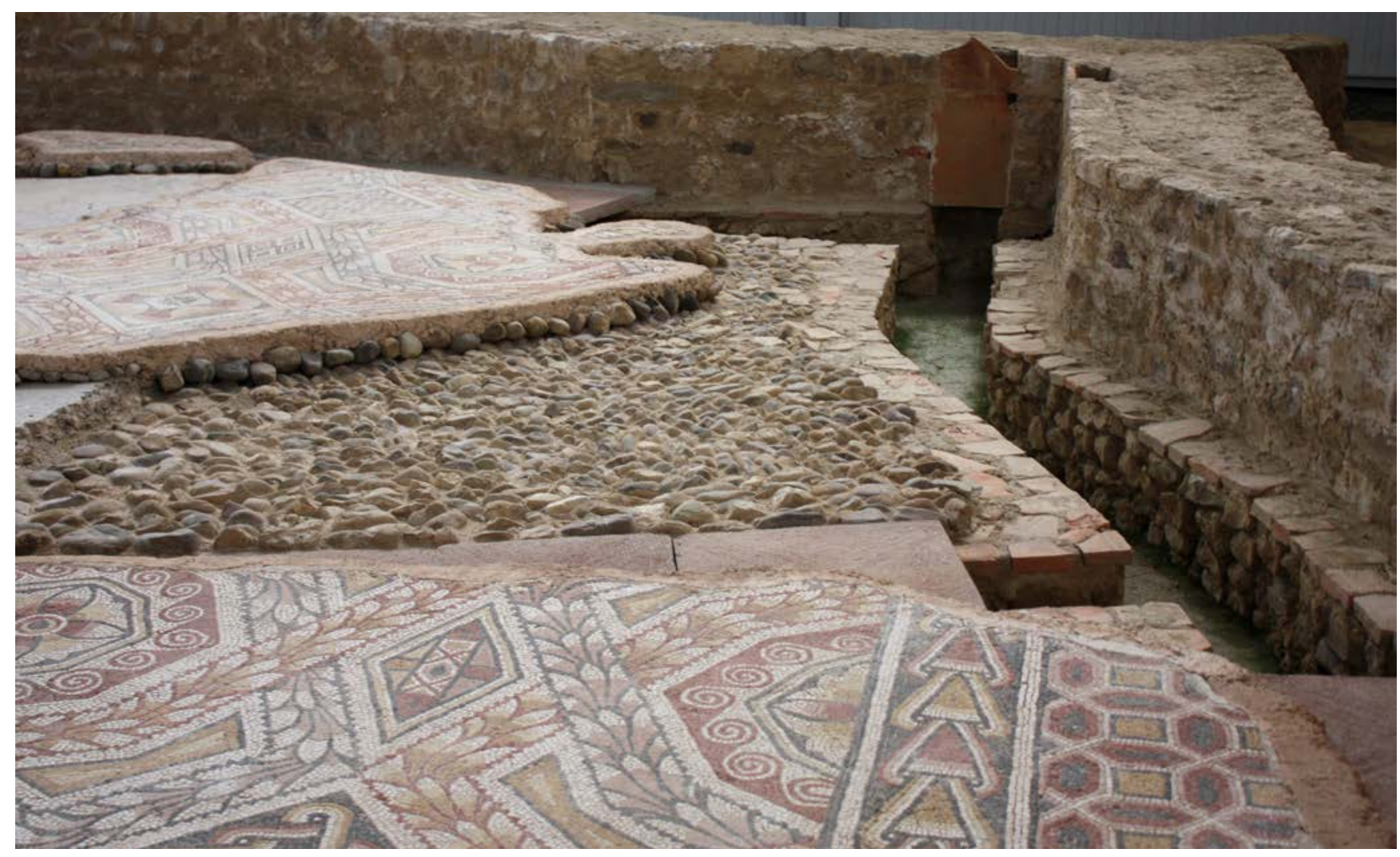

Figura 12. Restauración de conductos y pavimento, esquina suroriental (C. Gamarra). 
(19), por lo que en la última etapa de la villa el hypocaustum se puede suponer que no se utilizaba.

En las proximidades de los cuatro vértices del cuadrado, embutidos en los muros, se situaban las chimeneas de evacuación de los humos que ascenderían hasta la cubierta, siendo dobles en el lado este. Tienen dimensiones en torno a 20 x 20 cm e interiormente están tapadas con teja (figura 12).

Por lo que se refiere al mosaico hay que distinguir entre el gran cuadrado con la presencia de la yuxtaposición de parejas de hexágonos irregulares -escudos- y un gran emblema central con una estrella de ocho puntas, y los cuatro sectores circulares con dos diseños diferentes basados en el juego de cuadrados y hexágonos. El diseño del mosaico de escudos es frecuente en La Olmeda hallándose en algunas de las piezas más destacadas de la villa, como las galerías este y oeste del peristilo, además del apoditerio (dónde además coincide en el emblema central) (figuras 11-12).

\section{DISCUSIÓN}

El hypocaustum de V-16 realizado con pilae en todo el ámbito rectangular fue concebido y construido tal y como lo conocemos desde el momento inicial de esta cámara. Que el sistema constructivo del ámbito absidal fuera diferente pudo deberse únicamente a que la curvatura de los muros hacía más práctico y aconsejable el sistema de conductos. En todo caso su menor tamaño apenas modificaba las aceptables condiciones de acondicionamiento del conjunto. Todo indica que fue el primer hypocaustum de La Olmeda porque las características del mosaico de la habitación situado encima señalan que perteneció a la primera fase de mosaicos de la villa (20), encontrándose además situada en la zona residencial y junto al oecus.

El hypocaustum de la habitación V-32 comenzó a funcionar con la zona oriental del sector I que dispone de una única geometría de pilae, y los dos canales del sector II. En toda esa zona se dispuso un nuevo pavimento de mosaico que era notablemente diferente y de menos calidad que el resto de la zona rectangular (21). Es curioso, aunque necesario por cuestiones operativas del funcionamiento del hypocaustum, que ya en esta primera fase éste cuenta con los dos sectores.

En la zona ocupada por los dos conductos (sector II) no se construyó el típico hypocaustum de pilae porque la habitación existente debía contar con un pavimento de opus signinum que hubiera sido necesario demoler previamente. Por tanto, es muy posible que por ahorrar costes se hicieran solo los dos pequeños conductos longitudinales de $14 \times 40 \mathrm{~cm}$ a lo largo de esta cámara que a partir de ese momento se incorporaba al triclinio; las conducciones debían terminar en el muro colindante con la V-31, donde dos chimeneas evacuarían los humos hacia el exterior por encima de la cubierta.

En una fase posterior, coincidiendo con las obras del centrado de la última exedra se amplía el sector I del hypocaustum hacía el oeste lo que se manifiesta en el signo distintivo que representan las dos geometrías de pilae que se han expuesto.

El sector I del hypocaustum es el más próximo al praefurnium y el clásico de una cámara de fuego por lo que sería la zona más calurosa de la habitación que curiosamente coincide con un área básicamente pavimentado con opus signinum, por lo que tendría un uso secundario o residual frente a la mayor parte de la habitación pavimentada con mosaico (área señalada como "C" en la figura 6).

Como se ha mencionado, la ampliación del triclinio y la construcción del hypocaustum debían ser los objetivos de las trasformaciones realizadas en el comedor. Aunque a juzgar por una gran mancha de brasero en una zona relativamente central del mosaico hay dudas de la eficacia final del sistema calefactor (22). En cualquier caso la superficie ocupada por el hypocaustum de esta habitación es relativamente reducida sobre todo si se compara con el de V-16 que tendría por tanto unas mejores prestaciones.

Las características y el diseño del hypocaustum de B-12 son notablemente sorprendentes. La existencia de canalizaciones lineales y sobre todo su distribución un tanto irregular podrían indicar que la habitación estuvo previamente en uso a la ejecución del sistema calefactor.

De hecho, la habitación ya contaba con un solado de opus signinum en el momento en que se decide la ejecución de las obras del hypocaustum. Es decir, en un momento determinado del uso de la villa se realiza una reforma del salón existente que proporcione unos acabados y unas instalaciones que magnifiquen la categoría del mismo para mayor gloria de su propietario.

Hemos mencionado el magnífico mosaico geométrico que se coloca en esta habitación casi idéntico al primero que tuvo el apoditerio colindante B-10, así como a las galerías este y oeste del peristilo. La ejecución de un hypocaustum de canales supuso picar y levantar parcialmente el pavimento existente de opus signinum lo que podría justificar en cierto modo su geometría irregular. La operación resultaba más práctica y asequible que demoler todo el piso de la habitación para hacer un hypocaustum de una gran cámara con pilae y suspensura. Aunque la mencionada ejecución de los trabajos supone que la calidad o las prestaciones hipotéticas de uno y otro no serían iguales. De hecho se estima que el funcionamiento de este hypocaustum pudo ser cuando menos problemático y tal vez por ello no cumpliendo las expectativas se terminó por tapar la boca del praefurnium, que es tal y como se ha encontrado en las excavaciones.

La construcción de este hypocaustum se sitúa en algún punto intermedio entre los dos anteriores, pero posiblemente más cercano en el tiempo al primero que al segundo. De acuerdo con lo expuesto, la habitación estaba en uso cuando se decidió la construcción del hypocaustum, por cuanto disponía del pavimento de opus signinum. Se levantó parcialmente ese pavimento para hacer los canales y posteriormente se dispuso un mosaico casi idéntico a otros de la villa que se corresponden con los de la primera fase. Es muy posible que fuera de los últimos de esa primera fase que debió desarrollarse durante bastantes años.

Los materiales constructivos empleados en las tres habitaciones son diferentes lo que evidentemente también refuerza la idea de que fueron ejecutados en distintas épocas, según las necesidades a lo largo de la evolución de la villa. En el hypocaustum de la habitación V-16 los pilae se construyen con ladrillos pedales, mientas en la habitación V-32 se utilizan bessalis y en las paredes de los canales de la B-12 se encuentran adobes y piedra, y no solamente ladrillos, como en las otras habitaciones. Para la suspensura en los canales de la habitación B-12 se han encontrado ladrillos bipedales 
mientras que en las otras habitaciones sobre los pilae se han hallado dobles placas de tegulae o de ladrillo de otras dimensiones.

\section{CONCLUSIONES}

Los hipocaustos de las tres habitaciones son muy diferentes. Aunque formalmente se pueden agrupar dentro de los dos característicos de este sistema: el primero, el clásico a base de una gran cavidad con los pilae que soportan la suspensura, y el segundo formado por varios canales lineales comunicados, ya sean rectos o curvos.

Las diferencias entre unos y otros indican claramente que fueron construidos en distintas épocas y con condicionantes diversos. Se podría decir que el hypocaustum de V-16 es el único de los estudiados que fue planificado con antelación al uso previsto para esta habitación. Y quizás por eso el sector principal que ocupa la mayor parte de la cámara se construyó con el hypocaustum romano más típico a base de pilae y suspensura. En ese sentido, sería el más antiguo de la villa y el único proyectado previamente a su construcción. Los otros dos hypocausta corresponden a reformas posteriores al primer uso. El hypocaustum de la habitación B-12 debió ser el segundo porque aunque se halla en la zona termal que ya de por sí es una reforma del hipotético proyecto inicial, el mosaico debió ser de los últimos de la primera generación, mientras que los mosaicos sobre el hypocaustum de la habitación V-32 son claramente de una segunda fase.

El hypocaustum del triclinio V-32 fue construido en varias fases, todas ellas previsiblemente posteriores a la puesta en servicio de la estancia. Del análisis del pavimento, los muros y del propio hypocaustum se deduce que en un momento determinado el propietario tomó la decisión de ampliar el comedor y dotarlo de un sistema calefactor. De este modo solo disponía de hypocaustum la zona ampliada, con dos sectores diferentes que aluden claramente a unos condicionantes previos en su construcción, así como a su desarrollo en varias etapas.

El sector II a base de dos canalizaciones se realizó horadando un pavimento previo de opus signinum, mientras que el sec- tor I, construido al modo clásico, se realizó sobre un espacio exterior sin ninguna interferencia con elementos constructivos. Incluso en este último sector (I) se detectan dos épocas que se explican por la forma de la planta del hypocaustum, la orientación de los pilae, los mosaicos conservados de la exedra o el pavimento de opus signinum o mosaico sobre el mismo.

El hipocausto del salón de baños B-12 también debió construirse con posterioridad al primer uso de la habitación. Es decir que ya disponía de un pavimento de opus signinum que se levantó parcialmente para construir y excavar en la arcilla unos canales de una geometría relativamente imperfecta. Se trataba de ennoblecer y dotar de sistema calefactor.

Los hypocausta fueron construidos con materiales diversos. Entre estos materiales el más utilizado es el cerámico con piezas de diferentes formas y dimensiones. Por un lado los pilae de ladrillo, por otro los canales con paredes de piedra caliza, adobe o ladrillo, o de diferente tipología de suspensura, dobles placas de tegulae o ladrillo sobre los pilae, o bipedales sobre los canales, lo que refuerza evidentemente la idea de que los hypocausta se construyeron en diferentes épocas y por distintos maestros o técnicos de la construcción.

Como resumen se podría decir que del análisis de los hypocausta de La Olmeda se llega a la conclusión de que cuando no había preexistencias o elementos constructivos previos se optaba por el sistema clásico de una única cámara de gases sobre pilae que siempre era una mejor garantía de funcionamiento, como en la V-16 o en parte de la V-32. Y en el caso de reformas con condicionantes constructivos se optaba por la apertura de canalizaciones en la losa del suelo existente pero reaprovechando la mayor parte de ese elemento, como en la B-12 y en el resto de la V-32.

\section{AGRADECIMIENTOS}

Al Servicio de Cultura de la Diputación de Palencia por haber permitido acceder al interior de las estancias para realizar la investigación.

\section{REFERENCIAS}

(1) Gamarra, C. (2015). La Villa romana de La Olmeda. El Proyecto Arquitectónico (Tesis doctoral no publicada). Valladolid: Universidad de Valladolid.

(2) García Entero, V. (2005). Las transformaciones de los balnea rurales domésticos durante la Antigüedad Tardía en Hispania (ss. IV-VI). Cuadernos de Prehistoria y Arqueología, 32.

(3) Tsiolis, Vasilis. (2001). Las Termas de Fregellae: arquitectura, tecnología y cultura balnear en el Lacio durante los siglos III y II a.C. CuPAUAM: Cuadernos de Prehistoria y Arqueología 27: 85-114. P 104.

(4) Regueras Grande, F. (2013). Villas Romanas del Duero. Historia de un paisaje olvidado. Valladolid, Asociación Domvs Pvcelae. P 52.

(5) Graciani, A. (2009, mayo). Earthenware Pieces Manufactured for Roman Thermae. In Proceedings of the Third International Congress on Construction History (pp. 721-728). Cotbuss

(6) Guitart Trulls, Benito (1925). Termas, "hypocausta” y baños romanos en Mérida. Arquitectura, año VII num 76: 177-188

(7) Vitrubio, M. (1995) De Architectura. Madrid: Alianza Editorial (Traducción: José Luis Oliver Domingo) http://aparejadoresacc.com/wp-content/uploads/Vitruvio_Polion_Marco.pdf. Libro quinto, capítulo décimo.

(8) Guitart Trulls, Benito (1925). Termas, "hypocausta” y baños romanos en Mérida. Arquitectura, año VII, 76: 177-188.

(9) Font Arellano, J. (2005, 27 de enero). De Cancho Roano a La Olmeda: Mil años de construcción con tierra en la Hispania preislámica. Actas del Cuarto Congreso Nacional de Historia de la Construcción (pp 389-393). Cádiz: Instituto Juan de Herrera, SEHC, COAC, CAATC

(10) García Entero, V. (2001). Los balnea de las villae hispanorromanas. Provincia Tarraconense. Monografías de arquitectura romana, 5. P 336. 
(11) Carrillo, J. C. (1992).Técnicas Constructivas en las Villas Romanas de Andalucía. Grupo de Investigación HUM-236. $A A C, 3,309-339$.

(12) Palol, P. (1988). La villa romana de La Olmeda. Pedrosa de la Vega (Palencia). Guía de las Excavaciones. Palencia.

(13) Abásolo, J. A. Martínez, R. (2012). Villa Romana de La Olmeda, Guía Arqueológica, Diputación de Palencia.

(14) Abásolo, J. A. (2013). Los Mosaicos de La Olmeda, Lujo y ostentación de una villa romana. Diputación de Palencia.

(15) García Entero, V. (2001). Los balnea de las villae hispanorromanas. Provincia Tarraconense. Monografías de arquitectura romana, 5: P 338.

(16) Cortés, J., Ríos, D. (1988). El Hipocaustum de la habitación n ${ }^{0} 3$ de la villa romana de La Olmeda, Publicaciones de la Institución Tello Téllez de Meneses, $\mathrm{n}^{0}$ 58. Palencia, p. 10.

(17) Nozal, M., Ríos, D. (1997). Consolidación y restauración en un ámbito termal: la habitación nº 34 de la Villa Romana de la Olmeda. Publicaciones de la Institución Tello Téllez de Meneses, (68): 129-160. P 139.

(18) Nozal, M., Ríos, D. (1997). Consolidación y restauración en un ámbito termal: la habitación nº 34 de la Villa Romana de la Olmeda. Publicaciones de la Institución Tello Téllez de Meneses, (68): 129-160. P 150.

(19) Nozal, M., Ríos, D. (1997). Consolidación y restauración en un ámbito termal: la habitación nº 34 de la Villa Romana de la Olmeda. Publicaciones de la Institución Tello Téllez de Meneses, (68): 129-160. Pp 139-140.

(20) Cortés Alvárez de Miranda, R. (2008) Mosaicos en la villa romana La Olmeda. Diputación de Palencia.

(21) Abásolo, J. A., Martínez, R. (2012) Villa Romana de La Olmeda, Guía Arqueológica. Diputación de Palencia.

(22) Abásolo, J. A. (2013). Los Mosaicos de La Olmeda, Lujo y ostentación de una villa romana. Diputación de Palencia. 